\title{
TOWARDS POST-DISASTER DEBRIS IDENTIFICATION FOR PRECISE DAMAGE AND RECOVERY ASSESSMENTS FROM UAV AND SATELLITE IMAGES
}

\author{
S. Ghaffarian*, N. Kerle
}

University of Twente, Faculty of Geo-Information Science and Earth Observation (ITC), 7500 AE, Enschede, The Netherlands s.ghaffarian,n.kerle@utwente.nl

KEY WORDS: Debris identification, damage assessment, recovery, UAV, satellite images.

\begin{abstract}
:
Often disasters cause structural damages and produce rubble and debris, depending on their magnitude and type. The initial disaster response activity is evaluation of the damages, i.e. creation of a detailed damage estimation for different object types throughout the affected area. First responders and government stakeholders require the damage information to plan rescue operations and later on to guide the recovery process. Remote sensing, due to its agile data acquisition capability, synoptic coverage and low cost, has long been used as a vital tool to collect information after a disaster and conduct damage assessment. To detect damages from remote sensing imagery (both UAV and satellite images) structural rubble/debris has been employed as a proxy to detect damaged buildings/areas. However, disaster debris often includes vegetation, sediments and relocated personal property in addition to structural rubble, i.e. items that are wind- or waterborne and not necessarily associated with the closest building. Traditionally, land cover classification-based damage detection has been categorizing debris as damaged areas. However, in particular in waterborne disaster such as tsunamis or storm surges, vast areas end up being debris covered, effectively hindering actual building damage to be detected, and leading to an overestimation of damaged area. Therefore, to perform a precise damage assessment, and consequently recovery assessment that relies on a clear damage benchmark, it is crucial to separate actual structural rubble from ephemeral debris. In this study two approaches were investigated for two types of data (i.e., UAV images, and multi-temporal satellite images). To do so, three textural analysis, i.e., Gabor filters, Local Binary Pattern (LBP), and Histogram of the Oriented Gradients (HOG), were implemented on mosaic UAV images, and the relation between debris type and their time of removal was investigated using very high-resolution satellite images. The results showed that the HOG features, among other texture features, have the potential to be used for debris identification. In addition, multi-temporal satellite image analysis showed that debris removal time needs to be investigated using daily images, because the removal time of debris may change based on the type of disaster and its location.
\end{abstract}

\section{INTRODUCTION}

Building/structural damage assessment is a crucial task at the first stage for post-disaster response phase by supporting rescue operations, and then recovery phase by providing key information to support governments and decision makers planning for reconstructions.

Remote sensing has been demonstrated as an essential and efficient tool for a rapid damage assessment after a disaster (Brunner et al. 2010). In addition, advances in computer vision and photogrammetry allow scientists to develop complicated but advanced methods. Several studies were conducted for damage assessment using remote sensing data such as UAV (Cotrufo et al. 2018; Galarreta et al. 2015; Vetrivel et al. 2017; Vetrivel et al. 2016) and satellite images (Duarte et al. 2018b; Gillespie et al. 2007; Joshi et al. 2017). Most of them are based on the assumption that the urban disaster debris belongs to building rubbles, and the presence of the debris surrounding and inside/on the buildings has been used as a proxy to extract damage ratio (Kerle and Hoffman 2013). For example, Galarreta et al. (2015) used rubble piles as a damage feature to identify the damage ratio to the buildings from 3D point clouds derived from UAV images. Vetrivel et al. (2015) detected rubble piles/debris around/on/inside buildings in addition to gaps to extract damaged regions of the structures. In another study, Vetrivel et al. (2016) developed a method to detect building damage corresponding to debris, rubble piles and heavy spalling buildings. In addition, Ural et al. (2011) developed a method for larger urban area damage extraction using very high-resolution satellite images and LiDAR data and showed the efficiency of their method in extracting damaged buildings and their footprint. For a precise damage detection (Duarte et al. 2018a; Duarte et al. 2018b) fused satellite and UAV images using a CNN-based approach and improved the accuracy of the rubble/debris-based damage identification results. All of the aforementioned studies extracted the building damages with a high success/accuracy rate via mainly extracting the rubble piles/debris. However, disaster debris often incorporates sediments, vegetative debris, and personal property in addition to building materials/rubble, which is not necessarily belong to the closest building. For example, in a tsunami/storm surge scenario a large quantity of mixed debris can be washed up close to the intact buildings or a high-speed wind can rip roofs off houses and pluck tree fronds, and relocate them during the event. Furthermore, to do a post-disaster recovery assessment, damage assessment is needed as the first step to determine the damaged areas and ratios. Changes in land cover and land use of the areas were mostly used for damage and consequently recovery assessment of the areas (Ghaffarian et al. 2018; Ishihara and Tadono 2017). Hence, land cover/use classification/change detection of post-disaster satellite images the debris class is mostly used as an indicator/proxy of the damaged building and roads/area (Ghaffarian et al. 2018). For example, land cover-based change detection derived from highresolution satellite images was used for recovery assessment by Brown et al. (2010); Brown et al. (2008). They showed the

\footnotetext{
* Corresponding author
} 


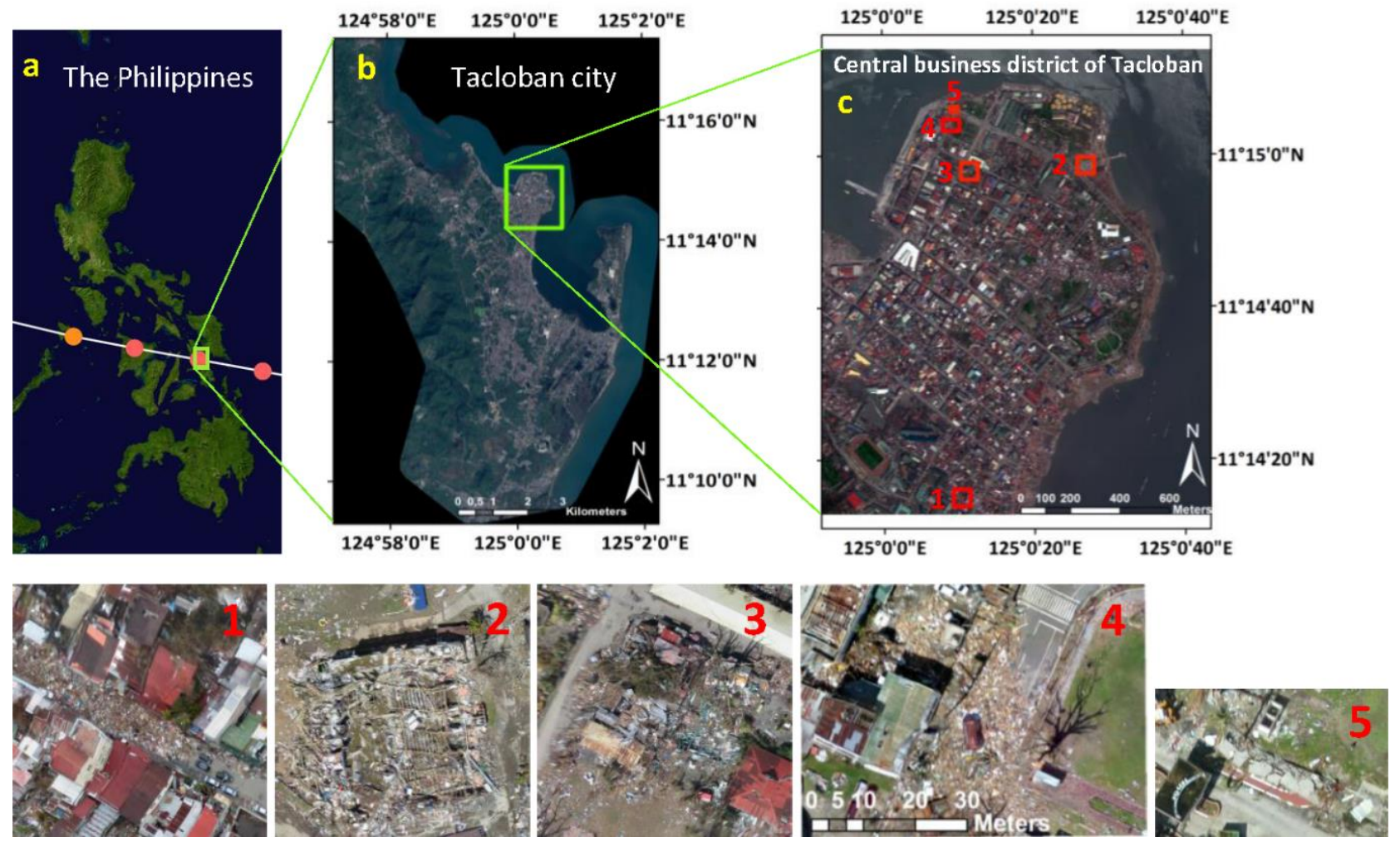

Figure 1. a) Track of Typhoon Haiyan over the Philippines, b-c) Overview of Tacloban city. 1-5) The selected UAV images for one week after disaster from the study area.

potential of using land cover change monitoring from satellite images in large area damage assessment and recovery mapping. In another study, Sheykhmousa et al. (2018) studied the land cover and land use changes for recovery assessment using very high-resolution satellite imagers and conducted a land cover and land use assessments for a few days after a disaster. They demonstrated that the remobilized debris in the entire area caused inaccuracies in land cover and land use classification results. Land cover and land use classification of the damaged area, particularly in water-related disasters, are prone to overestimation due to washed-up debris that may hinder the intact road and structures and also lead to overestimation of the post-disaster damaged area/ratio. Hence, identification of the disaster debris types is critical for precise post-disaster damage and consequently recovery assessments from both UAV and satellite images.

In this study, we aim to address the challenge of post-disaster debris identification by investigating the potential of the $\mathrm{UAV} /$ drone images and multi-temporal very high-resolution satellite images acquired some days, weeks and months after a disaster to distinguish between quasi-permanent debris (e.g., rubble related to building materials) and ephemeral materials that get continuously remobilized (e.g. flotsam deposited by flood/storm surge water, and wind-blown vegetation matter such as palm fronds).

\section{METHODOLOGY}

Two distinct approaches are proposed in this paper to identify the debris types for after disaster situation. UAV images due to providing very high-resolution images can contribute to the identification of the disaster debris types. Thus, we conducted textural analysis using the UAV images to compare areas with quasi-permanent debris and ephemeral materials and figure out the most informative ones to distinguish them. In the second approach, the idea of that the ephemeral materials can be collected much earlier than the structural debris is investigated to identify the debris types from multi-temporal satellite images.

\subsection{Textural analysis}

In order to distinguish the quasi-permanent structural rubble from other more remobilized debris from UAV images three textural analysis were investigated, i.e. Local Binary Patterns (LBPs), Gabor features, and Histogram of the Oriented Gradients (HOG).

\section{Texture features (LBPs and Gabor features)}

In general, image-based textural features are extracted using two approaches; statistical-based and signal processing-based approaches. Statistical methods make use of statistical relations of the spatial distribution of gray-level brightness values within the image. Currently, gray level co-occurrence matrix (GLCM) is one of the well-known statistical-based methods for textural analysis, which is also used as a basis for other advanced textural methods (e.g., Local Binary Pattern). GLCM features are used for different remote sensing application such as land use classification (Kabir et al. 2010; Pacifici et al. 2009), slum area detection (Kuffer et al. 2016b), built-up area extraction (Pesaresi et al. 2008), and high resolution satellite image analysis (Zhang et al. 2017). GLCM-based Local Binary Patterns (LBP) have been indicated as one of the most useful and powerful texture analysing for high-resolution remote sensing images due to their computational simplicity and discriminative power (Gevaert et al. 2016; Kuffer et al. 2016a; Mboga et al. 2017).

GLCM features (e.g., entropy, mean, correlation, homogeneity) are computed based on the occurrence of a pair of grey-level pixels in an image in predefined directions (Rao et al. 2002). Local Binary Pattern (LBP) features (Ojala et al. 2002) are computed based on a selected number of neighboring pixels $(\mathrm{N})$ 
at a defined distance (d) from the central pixel, which is rotationally invariant. LBPs are developed to identify uniform features, such as corners and edges. In this study, LBP features were extracted with $\mathrm{N}=8$ and $\mathrm{d}=16$ from UAV images.

Signal processing-based texture extraction approaches cut up image data into different frequency components [ref], and use frequency information of the signals in addition to spatial characteristics of the selected image. One of the well-known, such textural analysis is a wavelet-based method (Arivazhagan et al. 2006). Wavelet-based texture features were also used for remote sensing applications (Vetrivel et al. 2017) and found to be superior to GLCM texture features in many applications including classification of remote sensing images (Ruiz et al. 2004). Furthermore, wavelets and particularly one of its methods Gabor filters were used for damage assessment from remote sensing imagery (Radhika et al. 2012; Vetrivel et al. 2016). Gabor filters/features, which is based on wavelets, have been indicated as a robust texture extraction method for damage detection (Arivazhagan et al. 2006; Vetrivel et al. 2015, 2016). Gabor features are computed using a set of filters, and each of which is specifically defined to carry out frequency information at a specific orientation. Gabor filters separate image regions based on spatial frequency and orientation. Detailed information about generation of the Gabor filters and their application are given by Arivazhagan et al. (2006).

Both LBP and Gabor features are used in this study to investigate their usefulness in identifying debris types.

\section{Histogram of the Oriented Gradients (HOG)}

The HOG is a feature descriptor that is widely used in computer vision and remote sensing for object detection and classification (Dalal and Triggs 2005; Gao et al. 2013; Xiao et al. 2015; Xu and Liu 2016; Xu et al. 2016). The HOG uses spatial distribution of the gradients in the image regions to measure the spatial variation of edge orientations within a region (Kobayashi et al. 2008), which is a crucial factor in defining/extracting the shape of an object (Dalal and Triggs 2005). To extract HOG features, the magnitude and angle of the gradient of pixels in the image are computed. Then the magnitude of the gradients is binned into a histogram according to their angle/orientation for each predefined image block/cell. After normalizing the results of all blocks, they are concatenated to generate the image based on the block size. Furthermore, feature vectors that represent the gradient orientation and magnitude of blocks can be computed from the histograms. The HOG feature vectors were computed in this study from UAV images to investigate their utility in identifying debris types.

\subsection{Multi-temporal analysis}

Since structural rubbles are supposed to be heavier than ephemeral debris, they should be removed easier, and thus earlier, than structural rubbles after a disaster. Hence, the temporal change of the debris deposits in the images are monitored using multi-temporal satellite images. Furthermore, since the size of debris range from very small to bigger objects in the images, very high-resolution satellite images with 0.5 spatial resolution are employed.

\section{RESULTS AND DISCUSSIONS}

The proposed approaches were tested in Tacloban city, the Philippines, which hit by super Typhoon Haiyan on November 2013. Since the Typhoon Haiyan also caused a storm surge during the event, which led to deposit washed-up debris in urban areas, it is suitable to examine the proposed approaches. Five different locations of the debris were selected from the urban area of the Tacloban to implement the proposed approaches and discuss the results (Fig.1).

\subsection{Textural analysis of UAV images}

Three textural methods (i.e., HOG, LBP and Gabor filters/magnitude) were implemented on the five selected UAV image regions. From the selected images \#1 and \#4 mostly include ephemeral debris, while the others mostly consist of the regions with quasi-permanent structural rubbles.

The LBP and Gabor features are studied, and no significant difference between debris types are found.

Figure 2 shows the results of the HOG textural analysis of the selected UAV images. Visual inspection of the results shows a slight difference between HOG of the ephemeral debris and quasi-structural rubble, while ephemeral debris has spread distribution of the gradient orientations (see HOG of images 1 and 2). Structural rubbles due to containing bigger sized objects have less spread HOG distribution. However, visually inspection of the other textural results does not show a significant difference between debris types.
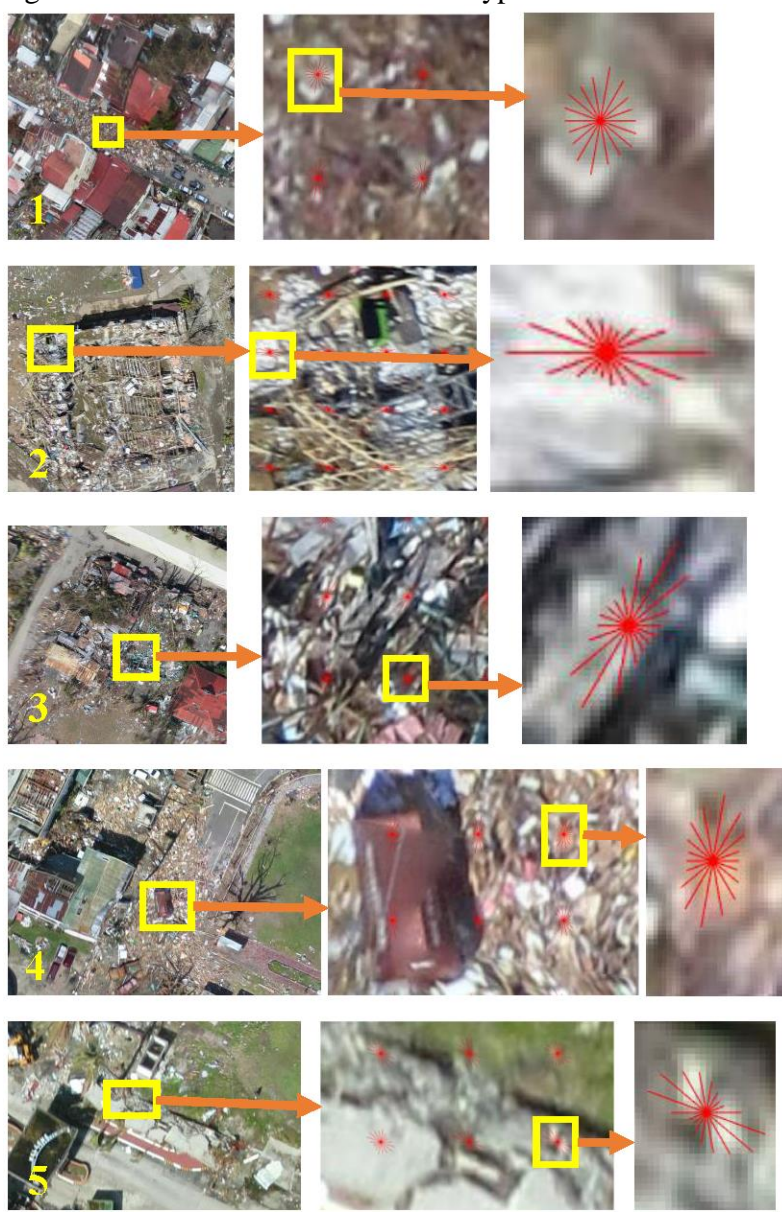

Figure 2. 1-5) UAV images and their corresponding HOG vector results for the denoted regions.

\subsection{Multi-temporal analysis of satellite images}

Satellite images acquired by different platforms (e.g., GeoEye1, World_View2 and 3, and Pleiades satellite) for 2, 3, 5, 7 days, $3,4,5$ weeks and 2,8 and 9 months after the disaster are analysed to detect debris changes and extract the relation of temporal changes with debris types in the area. 


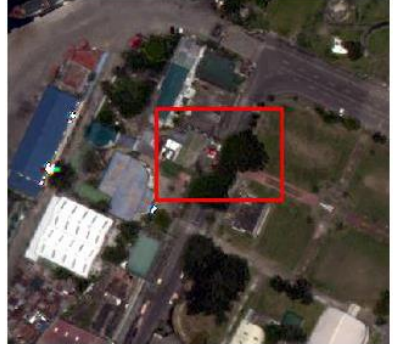

(a) Before the disaster

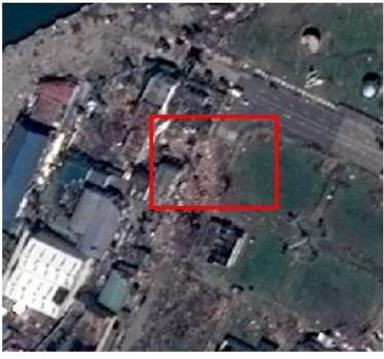

(e) one week after the disaster

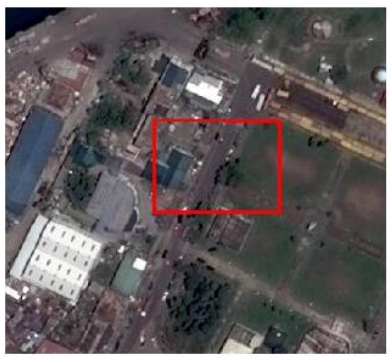

(i) 8 months after the disaster

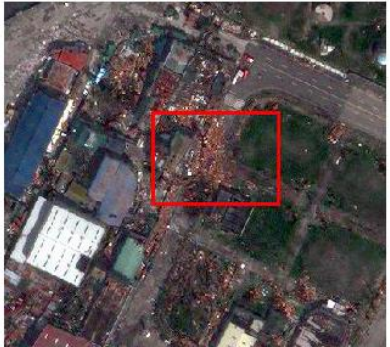

(b) 2 days after the disaster

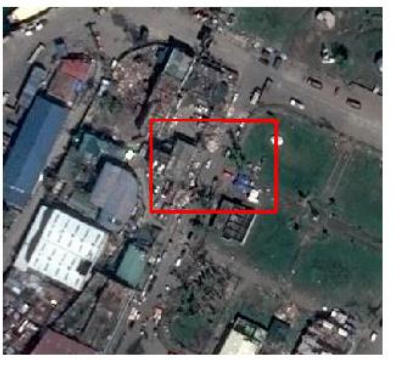

(f) 4 weeks after the disaster

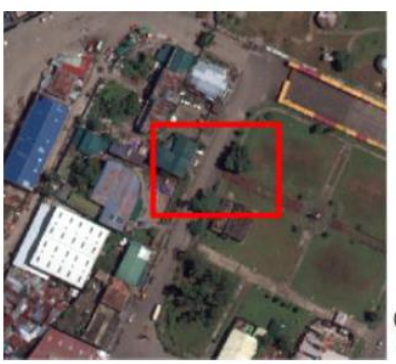

(j) 9 months after the disaster

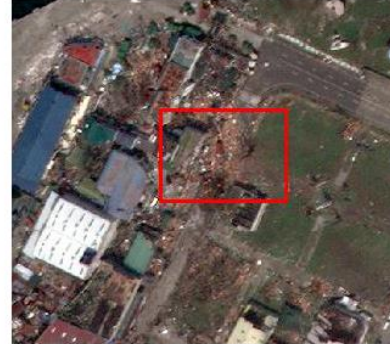

(c) 3 days after the disaster

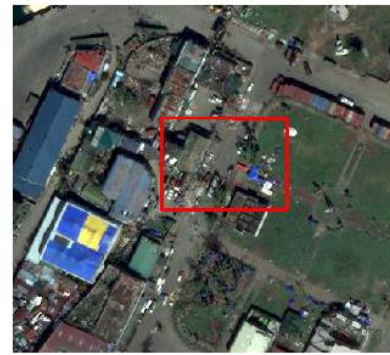

(g) 5 weeks after the disaster

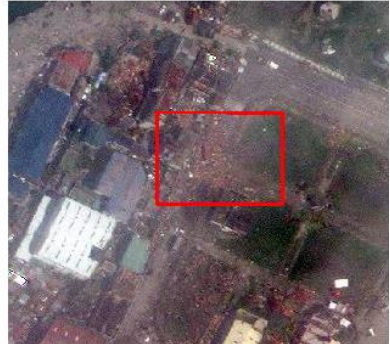

(d) 5 days after the disaster

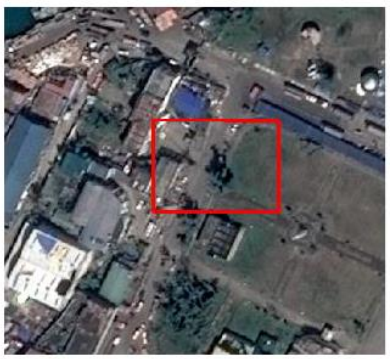

(h) 2 months after the disaster

Figure 3. a-j) Very high resolution satellite images respectively for before disaster, 2, 3, 5, 7 days, 4 and 5 weeks, and 2, 8 and 9 months after the disaster.

Figure 3 shows the satellite images for the selected area of Tacloban city. Considering the change in the debris area denoted at the images, we can see that most of the debris (on the road) was removed between the first week and 4 weeks after the disaster. However, remaining debris next to the road removed 5 weeks after the disaster. Since most of the debris had been disappeared from week 4 in the images, cannot find any relation between the removal time of the debris, and more satellite images are needed to study the changes between the first week and 4 weeks after the disaster.

\section{CONCLUSIONS AND FUTURE WORK}

In this paper, two approaches are proposed and analysed for post-disaster type identification. Textural methods are studied for UAV images to test HOG, LBP and Gabor features in differentiating the quasi-permanent structural rubble and ephemeral debris. The results showed that HOG is the most effective feature; however, in the future to precisely investigate the efficiency of the features and their effectiveness in disaster debris type identification they should be followed by a classification method and conduct a quantitative comparison. In addition, using 3D point clouds derived from UAV images will help debris identification. Furthermore, the idea of that ephemeral debris due to containing light weighted debris can be removed earlier than structural rubble is investigated using multi-temporal satellite images. Based on the achieved results in this study and the used time intervals after the disaster, we did not find a strong relationship between the time of removal and debris type. However, it is demonstrated that in order to study this approach we need daily data/images, type of the structures, and other characteristics of the considered areas that can influence the debris removal time and types. Hence, in the future, this idea should be studied using daily data (e.g., daily drone imagery), and use classification methods to extract the disaster debris areas quantitatively.

\section{ACKNOWLEDGEMENTS}

The GeoEye1, WorldView2 and WorldView3 satellite images have been granted by DigitalGlobe Foundation to the project. The Pleiades satellite images have been granted from European Space Agency (ESA) and Airbus Defence to the project.

\section{REFERENCES}

Arivazhagan, S., Ganesan, L., \& Priyal, S.P. (2006). Texture classification using Gabor wavelets based rotation invariant features. Pattern Recognition Letters, 27, 1976-1982

Brown, D., S., P., \& J., B. (2010). Disaster Recovery Indicators: guidelines for monitoring and evaluation. 
Brown, D., Saito, K., Spence, R., \& T., C. (2008). Indicators for measuring, monitoring and evaluating post-disaster recovery. In, 6th International Workshop on Remote Sensing for Disaster Applications

Brunner, D., Lemoine, G., \& Bruzzone, L. (2010). Earthquake Damage Assessment of Buildings Using VHR Optical and SAR Imagery. IEEE Transactions on Geoscience and Remote Sensing, 48, 2403-2420

Cotrufo, S., Sandu, C., Giulio Tonolo, F., \& Boccardo, P. (2018). Building damage assessment scale tailored to remote sensing vertical imagery. European Journal of Remote Sensing, 51,991-1005

Dalal, N., \& Triggs, B. (2005). Histograms of oriented gradients for human detection. In, 2005 IEEE Computer Society Conference on Computer Vision and Pattern Recognition (CVPR'05) (pp. 886-893 vol. 881)

Duarte, D., Nex, F., Kerle, N., \& Vosselman, G. (2018a). MultiResolution Feature Fusion for Image Classification of Building Damages with Convolutional Neural Networks. Remote Sensing, 10, 1636

Duarte, D., Nex, F., Kerle, N., \& Vosselman, G. (2018b). Satellite Image Classification of Building Damages Using Airborne and Satellite Image Samples in a Deep Learning Approach. ISPRS Annals of Photogrammetry, Remote Sensing and Spatial Information Sciences, IV-2, 89-96

Galarreta, J.F., Kerle, N., \& Gerke, M. (2015). UAV-based urban structural damage assessment using object-based image analysis and semantic reasoning. Natural Hazards and Earth System Sciences, 15, 1087-1101

Gao, F., Xu, Q., \& Li, B. (2013). Aircraft detection from VHR images based on circle-frequency filter and multilevel features. TheScientificWorldJournal, 2013, 917928-917928

Gevaert, C.M., Persello, C., Sliuzas, R., \& Vosselman, G. (2016). Classification of Informal Settlements through the Integration of $2 \mathrm{~d}$ and $3 \mathrm{~d}$ Features Extracted from Uav Data. ISPRS Annals of Photogrammetry, Remote Sensing and Spatial Information Sciences, III-3, 317-324

Ghaffarian, S., Kerle, N., \& Filatova, T. (2018). Remote Sensing-Based Proxies for Urban Disaster Risk Management and Resilience: A Review. Remote Sensing, 10, 1760

Gillespie, T.W., Chu, J., Frankenberg, E., \& Thomas, D. (2007). Assessment and Prediction of Natural Hazards from Satellite Imagery. Progress in Physical Geography, 31, 459-470

Ishihara, M., \& Tadono, T. (2017). Land cover changes induced by the great east Japan earthquake in 2011. Scientific Reports, 7,45769

Joshi, A.R., Tarte, I., Suresh, S., \& Koolagudi, S.G. (2017). Damage identification and assessment using image processing on post-disaster satellite imagery. In, 2017 IEEE Global Humanitarian Technology Conference (GHTC) (pp. 1-7)
Kabir, S., He, D.C., Sanusi, M.A., \& Wan Hussina, W.M.A. (2010). Texture analysis of IKONOS satellite imagery for urban land use and land cover classification. The Imaging Science Journal, 58, 163-170

Kerle, N., \& Hoffman, R.R. (2013). Collaborative damage mapping for emergency response: the role of Cognitive Systems Engineering. Natural Hazards and Earth System Science, 13, 97-113

Kobayashi, T., Hidaka, A., \& Kurita, T. (2008). Selection of Histograms of Oriented Gradients Features for Pedestrian Detection. In (pp. 598-607). Berlin, Heidelberg: Springer Berlin Heidelberg

Kuffer, M., Pfeffer, K., \& Sliuzas, R. (2016a). Slums from Space-15 Years of Slum Mapping Using Remote Sensing. Remote Sensing, 8, 455

Kuffer, M., Pfeffer, K., Sliuzas, R., \& Baud, I. (2016b). Extraction of Slum Areas From VHR Imagery Using GLCM Variance. Ieee Journal of Selected Topics in Applied Earth Observations and Remote Sensing, 9, 1830-1840

Mboga, N., Persello, C., Bergado, J., \& Stein, A. (2017). Detection of Informal Settlements from VHR Images Using Convolutional Neural Networks. Remote Sensing, 9, 1106

Ojala, T., Pietikainen, M., \& Maenpaa, T. (2002). Multiresolution gray-scale and rotation invariant texture classification with local binary patterns. IEEE Transactions on Pattern Analysis and Machine Intelligence, 24, 971-987

Pacifici, F., Chini, M., \& Emery, W.J. (2009). A neural network approach using multi-scale textural metrics from very highresolution panchromatic imagery for urban land-use classification. Remote Sensing of Environment, 113, 1276-1292

Pesaresi, M., Gerhardinger, A., \& Kayitakire, F. (2008). A Robust Built-Up Area Presence Index by Anisotropic RotationInvariant Textural Measure. Ieee Journal of Selected Topics in Applied Earth Observations and Remote Sensing, 1, 180-192

Radhika, S., Tamura, Y., \& Matsui, M. (2012). Use of poststorm images for automated tornado-borne debris path identification using texture-wavelet analysis. Journal of Wind Engineering and Industrial Aerodynamics, 107-108, 202-213

Rao, P.V.N., Sai, M.V.R.S., Sreenivas, K., Rao, M.V.K., Rao, B.R.M., Dwivedi, R.S., \& Venkataratnam, L. (2002). Textural analysis of IRS-1D panchromatic data for land cover classification. International Journal of Remote Sensing, 23, 3327-3345

Ruiz, L.A., Fernández-Sarría, A., \& Recio, J.A. (2004). Texture feature extraction for classification of remote sensing data using wavelet decomposition: A comparative study. In, International Archives of the Photogrammetry, Remote Sensing and Spatial Information Sciences (pp. 1109-1114). XXXV- B4

Sheykhmousa, M., Kerle, N., Kuffer, M., \& Ghaffarian, S. (2018). Understanding post disaster recovery through assessment of land cover and land uswe changes using remote sensing 
Ural, S., Hussain, E., Kim, K., Fu, C.-S., \& Shan, J. (2011). Building Extraction and Rubble Mapping for City Port-auPrince Post-2010 Earthquake with GeoEye-1 Imagery and Lidar Data. Photogrammetric Engineering \& Remote Sensing, 77, 1011-1023

Vetrivel, A., Gerke, M., Kerle, N., Nex, F., \& Vosselman, G. (2017). Disaster damage detection through synergistic use of deep learning and 3D point cloud features derived from very high resolution oblique aerial images, and multiple-kernellearning. ISPRS Journal of Photogrammetry and Remote Sensing

Vetrivel, A., Gerke, M., Kerle, N., \& Vosselman, G. (2015). Identification of damage in buildings based on gaps in 3D point clouds from very high resolution oblique airborne images. ISPRS Journal of Photogrammetry and Remote Sensing, 105, $61-78$

Vetrivel, A., Gerke, M., Kerle, N., \& Vosselman, G. (2016). Identification of Structurally Damaged Areas in Airborne Oblique Images Using a Visual-Bag-of-Words Approach. Remote Sensing, 8, 231

Xiao, Z., Liu, Q., Tang, G., \& Zhai, X. (2015). Elliptic Fourier transformation-based histograms of oriented gradients for rotationally invariant object detection in remote-sensing images. International Journal of Remote Sensing, 36, 618-644

Xu, F., \& Liu, J.-h. (2016). Ship detection and extraction using visual saliency and histogram of oriented gradient. Optoelectronics Letters, 12, 473-477

Xu, Y., Yu, G., Wang, Y., Wu, X., \& Ma, Y. (2016). A Hybrid Vehicle Detection Method Based on Viola-Jones and HOG + SVM from UAV Images. Sensors (Basel, Switzerland), 16, 1325

Zhang, X., Cui, J., Wang, W., \& Lin, C. (2017). A Study for Texture Feature Extraction of High-Resolution Satellite Images Based on a Direction Measure and Gray Level Co-Occurrence Matrix Fusion Algorithm. Sensors (Basel), 17 